\title{
Embedded System Architecture for Mobile Augmented Reality Sailor Assistance Case Study
}

\author{
Jean-Philippe Diguet ${ }^{1}$, Neil Bergmann ${ }^{2}$ and Jean-Christophe Morgère ${ }^{1}$ \\ ${ }^{1}$ Lab-STICC, CNRS, Université de Bretagne Sud / UEB, Lorient, France \\ ${ }^{2}$ School of Info. Tech. and Elec. Eng., The University of Queensland, Brisbane, Australia \\ \{jean-philippe.diguet, jean-christophe.morgere\}@univ-ubs.fr, n.bergmann@itee.uq.edu.au
}

Keywords: Embedded System Design, Augmented Reality, Hardware/Software Codesign, Reconfigurable Computing.

\begin{abstract}
With upcoming see-through displays new kinds of applications of Augmented Reality are emerging. However this also raises questions about the design of associated embedded systems that must be lightweight and handle object positioning, heterogeneous sensors, wireless communications as well as graphic computation. This paper studies the specific case of a promising Mobile AR processor, which is different from usual graphics applications. A complete architecture is described, designed and prototyped on FPGA. It includes hardware/software partitioning based on the analysis of application requirements. The specification of an original and flexible coprocessor is detailed. Choices as well as optimizations of algorithms are also described. Implementation results and performance evaluation show the relevancy of the proposed approach and demonstrate a new kind of architecture focused on object processing and optimized for the AR domain.
\end{abstract}

\section{INTRODUCTION}

Recent breakthroughs in the domain of wearable displays indicate that Augmented Reality (AR) systems will bring new applications in the near future. However, this also implies an emerging challenge regarding the design of low-cost, low-power systems to be embedded in see-through glasses. Indeed, most of the research work in related conferences (e.g. ISMAR), doesnt focus on embedded system design but on specific AR issues such as reality overlay or virtual object handling. The objectives of this work are firstly an in-depth study of application requirements for specific positioning and drawing of 3D objects on a seethough screen, according to the user vision of field. Secondly this is the design of suitable hardware architecture solutions based on upcoming FPGA ${ }^{1}$ technologies. More precisely we address a problem that can be defined with the two following questions:

1) How to position and draw a list of relatively simple $3 D$ objects composed of a set of polygons, which are defined by vertices with $3 D$ coordinates on a seethrough head mounted displays (HMD)?

2) How to efficiently integrate this solution on an FPGA-based reconfigurable architecture, while considering flexibility for various application and complexity contexts?

\footnotetext{
${ }^{1}$ Field Programmable Gate Arrays
}

The paper is organized as follows. In Section 2, we present our motivations for this research field and the target applications we consider. Section 3 gives an overview of the relevant technologies for AR, including architectures. Section 4 describes the main steps of our original approach. Our solution is based on the adaptation of previous positioning solutions to the application context, on algorithmic transformations and on a new architectural solution for object drawing. In Section 5, we present our hardware/software architecture. Our solution is flexible and optimized according to AR applications and algorithm choices. In Section 6 , implementation results and performances estimations are given and discussed. Finally we conclude and draw some overall insights.

\section{CASE OF SAILOR ASSISTANCE}

\subsection{Application Context}

AR by itself is not a new topic but many challenges remain unsolved, especially in mobile and outdoor contexts where field markers arent applicable and video-assisted model-based tracking is usually inefficient in real-life luminosity conditions. The proposed application set is based on the following observations. First, designers already have at their dis- 


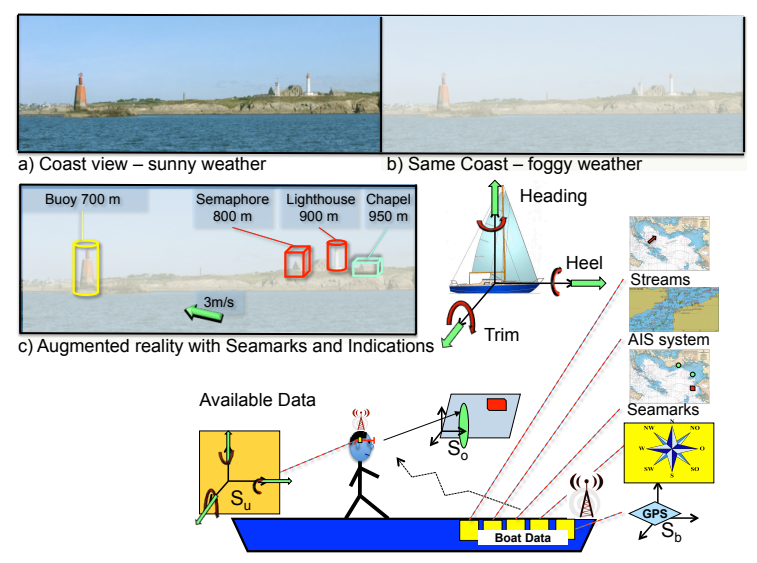

Figure 1: Sailor Assistance AR case study.

posal an ever-increasing amount of recorded and classified data of geolocalisation. Second, considering see-though glasses, these data can be added to a users field of vision to provide mobile outdoor AR applications. Third, 3D objects can be computed to fit with the landscape seen by the user if the user attitude can be obtained with appropriate sensors. Fourth, except for specific applications like architectural designs, the constraint of overlay accuracy is reduced for distant objects and no camera is required for the pose estimation. In this context many applications can be designed to improve security and orientation decisions, in different hands-free and low footprint devices. We consider the particular but complicated case study of sailor assistance from which can be derived various requirements for a generic system. On a boat, understanding of the position is vital when approaching sensitive environments such as coasts, open sea reefs or harbour navigation channels. These situations are true for small sailing or motorboats but also on large vessels, where the navigation crew is limited with respect to boat sizes. In these kinds of long ships, it is also recommended to combine visual checking, based on real environment observations, and instrument piloting. Current methods consist of going back and forth between map analysis and visual observations. Matching map indications with a real environment can be tricky and error-prone, and it also represents a loss of time that can be precious in case of emergency. Finally, matching can be simply impossible when the visibility is very bad (see Fig.1). This is a relevant case study since a ship is a very unstable system. All the continuing motions have various parameters depending on boat speed, user moves and ocean oscillations. Swell periods can vary between $0.05 \mathrm{~Hz}$ and $0.1 \mathrm{~Hz}$. But this is also a domain where a lot of data are available. The first category is composed of static seamarks objects, the second one is dynamic but can be estimated, for instance the ocean streams. The third one is related to the positions, heading and ID of boats or any maritime objects in the surroundings provided by the AIS system. All these data can be added to the user fields of vision according to position and attitude estimations. Then we have boatpositioning data, which include GPS measurements, speed, trim, heel, and heading. All these data can be obtained through a wireless network that doesnt require high bandwidth capacities. But while these data are useful they are not sufficient since it is necessary to know the user attitude defined with head angular positions. These data have to be provided by embedded sensors that must be integral with the glasses. Redundancy between boat and user data can also be usefully combined to improve accuracy. For instance the on-glasses accelerometers can be combined with the ship GPS to estimate local moves on a long vessel.

\subsection{Related Optimizations \& Challenges}

Specific optimizations can be applied with respect to general-purpose 3D graphics. We can point out three of them. i) Object distance means relaxed accuracy constraint: orientation information in outdoor applications is useful for distant objects. So one can relax the constraint of accuracy since the size of the object is decreasing with distance. It also means that a camera isnt required and neither are complex object tracking methods, which can be inefficient outdoors because of their sensitivity of luminosity changes. ii) No background and a limited number of objects: the background is the real world that can be seen with see through glasses, moreover ergonomics and obvious usefulness, impose that a limited number of objects can be drawn at the same time. iii) Static or slow objects: most useful orientation objects are static or move slowly if they are far away. All the previous features provide a rationale for a simplified implementation of 3D graphics that may be usefully exploited to optimize the design of the embedded system.

\section{STATE OF THE ART}

\subsection{MEMS Sensors}

The first breakthrough occurred in the domain of sensors for position, speed, acceleration and attitude (yaw, pitch, roll) measurements. For a long time, the size, and cost of such devices have limited their use to navigation instruments in aircrafts and satellites. However MEMS ${ }^{2}$ technologies are now provid-

\footnotetext{
${ }^{2}$ Micro Electro-Mechanical Systems
} 
ing integrated and low cost Inertial Measurement Unit (IMU) solutions (Li et al., 2008; Nasiri, 2010) that make possible the design of mobile consumer systems. The most widespread solution is based on the association of two kinds of MEMS devices: a 3-axis accelerometer sensor and a 3-axis magnetometer sensor. The combination of these sensors can provide the estimation of a body translations and attitude, which means 3 axes inclinations and so compass capabilities. More recently gyroscopes, that return angular velocities, have also been proposed in integrated versions. Invensense ${ }^{3}$ has unveiled in 2010 an IMU including a 3-axis integrated gyroscope (angular speed) combined with a 3-axis accelerometer. In September 2011 ST presented the iNemo ${ }^{4}$ engine that includes 3axis linear accelerometers, 3-axis angular speed measures, a magnetometer (heading) and a barometer (altitude). Like the Invensense solution, the whole device uses a 32 bit processor to run motion estimation algorithms. However it will be shown in Section 6 that a gyroscope is really not needed in our context, moreover we will see that a softcore synthesized on a FPGA can run motion estimation.

\subsection{HMD Displays}

The second kind of technology that opens new horizons to AR applications comes from the domain of HMD. New see-through glasses (see Fig.2), will soon be available. Some prototypes already exist and should soon be commercially available. Companies like Vuzix, Optinvent, Laster or Lumus have developed prototypes or already commercialize some products with important limitations. Google is also announcing glasses, which in reality seem to be a Head Mounted Display. HMDs are still very new, but this type of device paves the way to make future AR reality applications available at a reasonable cost. Moreover in 2011, the first prototype of a single-pixel lens has been demonstrated (Lingley et al., 2011). This now raises the question of integration of the embedded system since current approaches are based on wired connections with smart phones or laptops that provide data to this new display.

\subsection{Embedded System Architectures}

Miniaturization and power consumption are important issues for mobile AR systems, which mainly require computation resources for object positioning and for drawing, and also control capacities for data

\footnotetext{
${ }^{3}$ www.invensense.com/

${ }^{4}$ www.st.com/internet/evalboard/product/ 250367.jsp
}

acquisition from sensors with standard communication protocols. Different solutions may be considered for the implementation of such applications. The first solutions rely on advanced embedded multiprocessor architectures based on a CPU enhanced with a GPU. They are typically based on cortex ARM cores or Intel Atoms with specialized Graphics and Video coprocessors. The advantage of such architectures is the availability of software development frameworks. While high-resolution video games, video and image processing for object identification and online reality overlay would justify such impressive processing resources, for the types of AR applications we are targeting there is no need for cameras and complex pose computation including image processing, and so such CPU-GPU solutions would be overkill. Another possible solution is provided by reconfigurable architectures that enable specifically optimized and low frequency designs. These rely on Hardware / Software design methodologies and recent high-performance FPGAs. These FPGAs are often power-hungry however the roadmap of FPGAs is clearly focused on this power issue with the aim to address the embedded system market. The hybrid ARM/FPGA Zynq architecture, released by Xilinx in 2012, clearly opens new perspectives. On-chip memory capacity is also a key issue where significant progresses have been made. For example, the Artix Xilinx low power, low cost family embeds up to 12 Mbits of block RAMs. Regarding GPUs on FPGAs, Xylon has added a 3D graphics module to the Logibrick library. The architecture relies on a 3 stage pipeline: i) Geometry/Rasterization based on Micro-Blaze Xilinx softcore enhanced with a coprocessor able to compute coarse grain mathematical instructions; ii) Renderer: pixel color, texture, occlusion implemented as an accelerator connected to the PLB bus and iii) full rendered 3-D scene anti-aliasing also implemented as master hardware module. This solution is a simplified version of the usual graphics pipeline and is designed for general purpose Open-GL-ES applications. It shows that low frequency dedicated architectures can be designed for this purpose. In (Kingyens and Steffan, 2011) the authors present a GPU-inspired and multi-threaded softcore architecture, which is programmable with the NVIDIA Cg language. The aim is to simplify the use of FPGA-based acceleration boards for High Performance Computing. Our approach is different, strongly dedicated to embedded systems and AR applications with a high focus on data locality optimization for minimizing data transfers. From a general point of view, 3D graphics is a very complex and greedy application field but if we consider the most promising AR applications, we ob- 


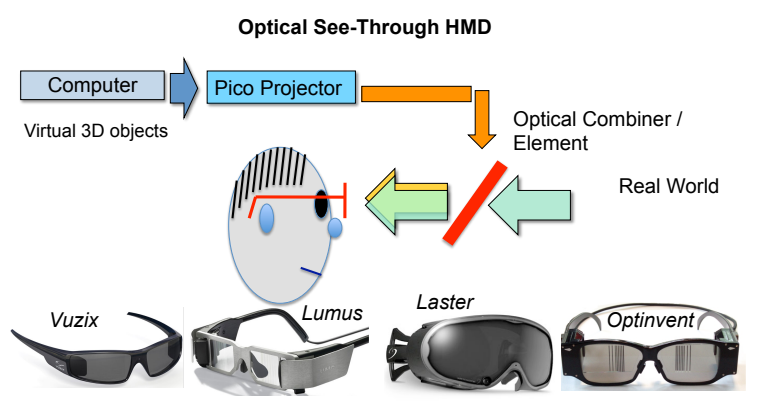

Figure 2: Upcoming see-through glasses.

serve that optimizations and simplifications are possible and can lead to very efficient solutions. Moreover, one can consider available soft cores that can run a standard Linux OS to simplify sensor interfaces. Such soft-cores have limited performance but can be enhanced with dedicated reconfigurable accelerators to implement energy-efficient flexible architectures on a single chip. As demonstrated in (Benkrid, 2010), FPGA can overcome CPU and GPU with orders of magnitude in terms of energy efficiency by allowing fully dedicated architectures. Finally dynamic hardware reconfiguration also provides opportunities to adapt the architecture to the application context and requirements. Our solution follows this approach.

\subsection{Conclusions}

AR research trends analysis shows that the five main research topics over the last ten years are Tracking techniques, Interaction techniques, AR applications, Calibration and registration and Display techniques. This state of the art is backed up by the first analysis and optimization opportunities given in Section 2.2. Significant progresses and research have been done on how to apply AR in terms of techniques and algorithms that are increasingly mature. AR has also pushed emerging display and sensor technologies but the question of integration has not been a major focus. People are more focused on the previously detailed issues and consider implementation and architecture as a secondary problem. By considering algorithms ready for our application domain our study is focussed on application / architecture matching.

\section{APPLICATION ALGORITHM CHOICES \& OPTIMIZATIONS}

\subsection{Solution Steps}

In the following Sections we present the design choices to specify and implement the complete appli- cation flow described in Fig.3. We address the three following points: i) Object positioning according to sensors and application context, ii) Object drawing, iii) some optimisations that have been introduced according to the application context.

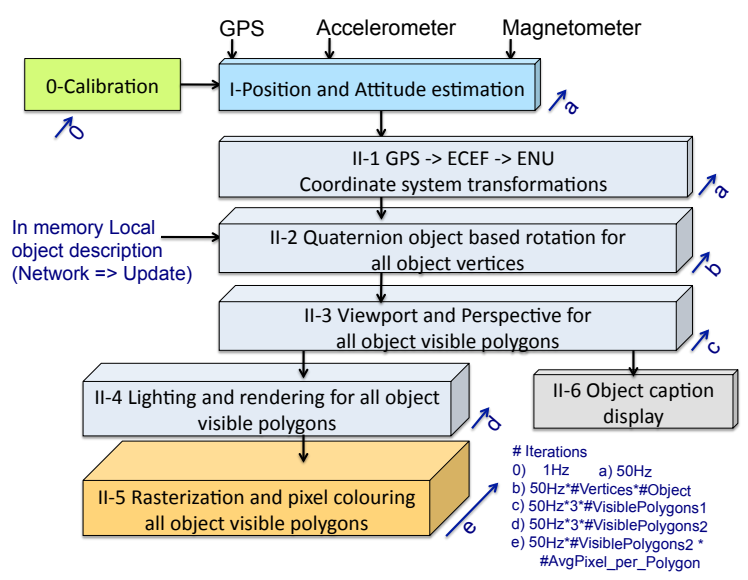

Figure 3: Global application flow.

\subsection{User Attitude Modelling}

In this domain the objective was to develop a robust and gyro-free solution based on magnetometer, GPS and accelerometer. There were two difficulties. The first one was the state of the art that was mainly related to aircraft, automotive systems or AR applications with computer-based implementation including gyroscopes and without great concerns about size and power consumption issues (Zhu et al., 2007; Waegli et al., 2007; Li et al., 2008). The second point is related to the filtering and estimation problem, actually determining position, speed and attitude from a set of noisy sensors is a non-linear problem that can't be solved with traditional Kalman filters. We've studied various kinds of alternative solutions for nonlinear filters, which were based on EKF (extended Kalman filter) (Kim et al., 2009), UKF (unscented Kalman filter) (Shin and El-Sheimy, 2004) or UPF (unscented particle filter) (Koo et al., 2009). According to the current project environment and constraints, it turned out that applying Wahbas method could solve the question of the gyroscope. This technique has been applied in aeronautics and avionics domain in (Gebre-Egziabher et al., 2000) and considers gravity and magnetic field as the two required noncollinear vectors. It is based on the quaternion modeling, which also offers interesting properties such as the computation complexity for rotations, the stability in presence of coding and rounding errors and the inherent robustness regarding the gimbal lock problem. The solution finally developed is based on a low com- 
plexity 6-states EKF algorithm for speed and position estimations. This EKF is loosely coupled with a low frequency GPS and gets the body attitude data, as a quaternion vector, from a 6-states KF algorithm that implements Wahba's method. The proposed EKF6 algorithm was previously applied in (Bijker and Steyn, 2008) with gyroscope data that are removed in the proposed version. Moreover the acceleration data are combined with data from GPS after filtering. The complete solution also relies on a robust method for the auto calibration of the magnetometer (Guo et al., 2008). It leads to a complex 14 states EKF algorithm, however it is used only once at start time or with a very low frequency if the environment is changing. Note that the solution can easily be augmented with new data. It means that if gyroscope data are available with reasonable cost and footprint, they can be added to the model. The complete solution for position and attitude estimation is described in Fig.4.

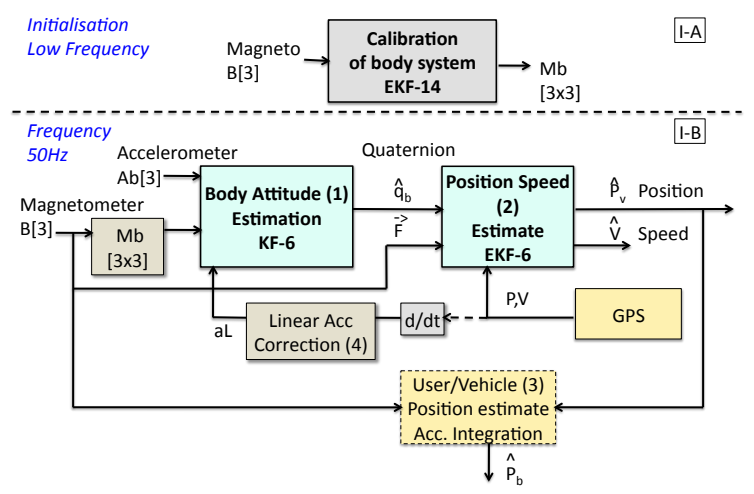

Figure 4: Position and attitude estimation.

\subsection{Virtual Object: Geometry Stage}

The objective is to select only objects of interest in the context of the user. The definition of interest is a real question that can be discussed but which is out of the scope of this paper. In this work we consider third configurable filters but various rules could be introduced. The first one is obvious; this is the position in the user field of view. The second is the minimum size of object after 2D projection. The third one is the choice of accessible objects stored in the object memory. This selection is implemented in software and can be configured according to confidentiality issues or contextual search criterions. Hereafter, we summarize the steps of the geometry stage.

\subsubsection{From GPS to ENU Coordinates}

Given the GPS coordinates, there are two successive transformations to apply: the first one from GPS to
ECEF (Earth-Centered, Earth-Fixed) and then from ECEF to ENU (East North Up).

\subsubsection{Object Rotation based on User Attitude}

The user attitude, namely the three angles that define user head orientation, is modeled with the quaternion formalism $\left(q=q_{0}+q_{1} \cdot i+q_{2} \cdot j+q_{3} \cdot k\right)$. Then a rotation (from $V$ to $V$ ) is applied to every point of every visible object according to the following operation:

$$
V=q \cdot V \cdot q^{-1} \text { where } q^{-1}=q_{0}-q_{1} \cdot i-q_{2} \cdot j-q_{3} \cdot k \text { (1) }
$$

\subsubsection{In-viewing Frustum Test, 3D $\rightarrow$ 2D}

The objective of the 2D projection step is firstly the selection of visible points and objects within the user field of vision and secondly the computation of 2D coordinates after a perspective projection. An object is considered as visible if it is in the field of vision, which is defined with horizontal and vertical angles and if it has a size bigger than a minimum sphere, which is defined by a ray $\mathrm{R}$. The viewport operation is based on these bounding values and requires, for every point of all relevant objects, 4 multiplications and 4 tests. Then the projection can be computed with 3 divisions per object and then 4 products for every point of all visible objects.

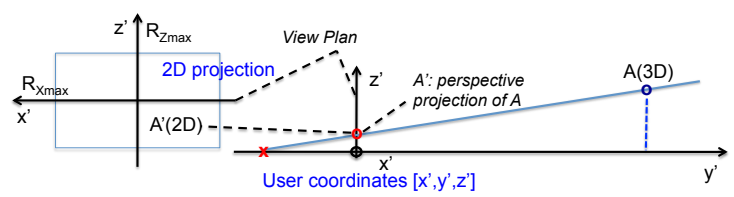

Figure 5: From user 3D coordinates to 2D view plan.

\subsection{Virtual Object Drawing}

\subsubsection{Visibility Tests}

Before drawing any object, three tests are applied. The first one has been described in the previous Section. The second test addresses the sign of polygon normal vectors. Considering opaque surfaces, if the $\mathrm{Y}$ value Ny of the polygon normal vector in the user coordinates system is negative, then the polygon isnt visible. Based on vertices coordinates, this value is computed for each polygon and requires 3 multiplications and 4 additions. The third test is the well-known Z-buffer test (Y-buffer with our conventions), the aim is to avoid drawing polygons, which are hidden by some closer ones. It is based on an array $\mathrm{A}[\mathrm{i}, \mathrm{j}]$ that stores the smaller $\mathrm{Y}$ value of the closer polygon point located at the address $(\mathrm{i}, \mathrm{j})$, where $\mathrm{i}$ and $\mathrm{j}$ correspond to the ith line and jth column of the display. The rasterization step computes the pixel coordinates of each 
point after the 2D projection (step (4) in Fig.3). This test can be implemented with a dedicated module that computes address and performs comparison and update, details are given in Section 6.

\subsubsection{Light Modelling and Optimization}

Models used are based on the barycentre method from OpenGL-ES. The light model for each Vertex of the triangle polygon is given in Fig. 6. Once the three vertex lights are computed $\left(L^{1}, L^{2}, L^{3}\right)$, the barycentre method can be applied to fill the triangle A,B,C and compute each vertex light as follows:

$$
L_{c}(x, z)=A(x, z) L_{c}^{1}+B(x, z) L_{c}^{2}+C(x, z) L_{c}^{3}
$$

where: $c=R, G, B, A(x, z)=F_{23}(x, z) / F_{23}\left(x_{1}, z_{1}\right)$; $B(x, z)=F_{31}(x, z) / F_{31}\left(x_{2}, z_{2}\right), \quad C(x, z)=$ $F_{12}(x, z) / F_{12}\left(x_{3}, z_{3}\right)$ and $F_{i j}(x, z)=\left(z_{i}-z_{j}\right) x+$ $\left(x_{j}-x_{i}\right) z+x_{i} \cdot z_{j}-x_{j} z_{i}$ The implementation of this method has been optimized as follows: $F_{i j}$ can be computed only once for each triangle and $K_{p x}(c)$ and $K_{p z}(c)$ can be defined as unique color increments on $\mathrm{X}$ and $\mathrm{Z}$ axis respectively: $K_{p x}(c)=L_{1}(c)\left(\left(z_{2}-z_{3}\right) / F_{23}\left(x_{1}, z_{1}\right)\right)+L_{2}(c)\left(\left(z_{3}-\right.\right.$ $\left.\left.z_{1}\right) / F_{31}\left(x_{2}, z_{2}\right)\right)+L_{3}(c)\left(\left(z_{1}-z_{2}\right) / F_{12}\left(x_{3}, z_{3}\right)\right)$ $K_{p z}(c)=L_{1}(c)\left(\left(x_{3}-x_{2}\right) / F_{23}\left(x_{1}, z_{1}\right)\right)+L_{2}(c)\left(\left(x_{1}-\right.\right.$ $\left.\left.x_{3}\right) / F_{31}\left(x_{2}, z_{2}\right)\right)+L_{3}(c)\left(\left(x_{2}-x_{1}\right) / F_{12}\left(x_{3}, z_{3}\right)\right)$ where $1 / F_{i j}$ is computed only once per polygon to remove divisions. Finally we obtain a simple algorithm based on fixed increments on both $\mathrm{X}$ and $\mathrm{Z}$ axis:

$$
\begin{aligned}
& L_{c}(x+1, z)=L_{c}(x, z)+K_{p x}(c) \\
& L_{c}(x, z+1)=L_{c}(x, z)+K_{p z}(c)
\end{aligned}
$$

This reorganization of computation has a strong impact on complexity since 3 DIV, 21 MULT and 12 $\mathrm{ADD}$ are required per visible polygon and then only 3 additions per point

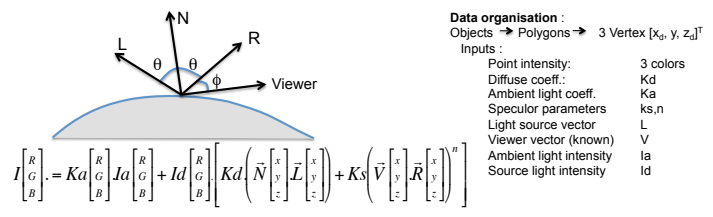

Figure 6: Light modelling.

\subsubsection{Scan-line and Incremental Pixel Shading}

The process of rasterization consists of mapping the real pixel addresses on the discrete display grid. The idea of the method is based on the use of the wellknown Bresenham algorithm for line drawing that eliminates divisions. In our particular case, we consider triangles so the algorithm first sorts out the three triangle vertices (yellow point in Fig.7) on the Xaxis, and then simultaneously draw two of the three segments (Pt1-Pt2 and Pt1-Pt3) and fill the triangle with $K_{p x}$ and $K_{p z}$ color increments (dotted lines: pixel colouring order). Once a triangle point is reached, the two remaining segment are considered and the same method is applied (Pt3-Pt2 and Pt3-Pt1). The main algorithm is the control of the pixel-shading method that also calls three key procedures. InitDrawPolygon() computes the 3 triangle vertex colors, InitCoeffPolygon() computes $K_{p x}(c)$ and $K_{p z}(c)$ color increments and IncDraw() writes the pixel value in the RGB format.

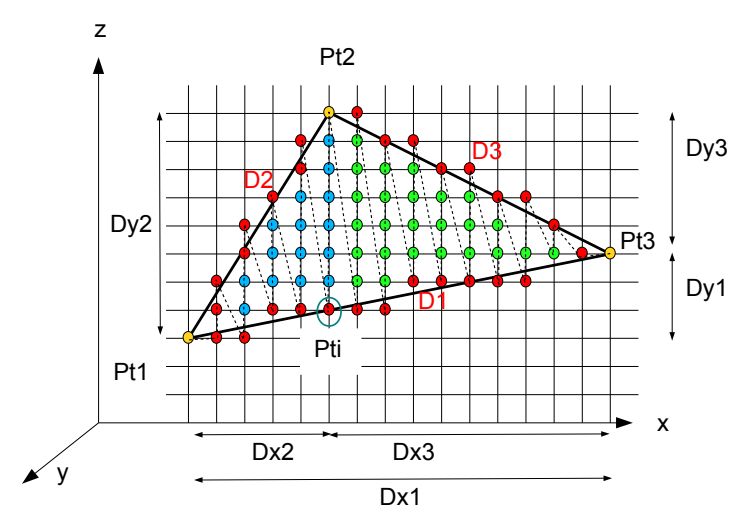

Figure 7: Incremental Pixel Shading principle

\section{EMBEDDED SYSTEM ARCHITECTURE}

Considering the mobile AR with distant and simple objects, the project objectives and the optimization opportunities, the first step was the analytical estimation of performances. The second one was a projection on various architecture models on Xilinx FPGA that led to partitioning between software (softcore or hardcore CPU) and optimized and dedicated hardware implementations. The next step was the specification of the heterogeneous architecture and VHDL coding at RTL-level. We will see in Section 6 that some hardware implementations were necessary. In this Section we detail the final heterogeneous architecture model.

\subsection{Multiple OP H-MPSoC}

The system architecture is fully specified and tested at a cycle level, the VHDL implementation is completed and synthesized, moreover HW / Linux interfaces have been specified. The architecture, obtained 
after specialization and hardware/software partitioning, is described in Fig. 10. The result is compact and flexible. The architecture is mainly built around a softcore MicroBlaze (MB) running Linux (Petalinux) to simplify the access to standard peripherals (I2C, UART, Ethernet) used for network access and communications with sensors. According to the specific AR context, the choice has been done to consider positioning and graphics at the object level. So, the processor is enhanced with some new graphic processors called OP that handle object positioning and drawing. Each OP is in charge of one or more objects. Video buffers are stored in DDR and memory access is implemented with the MPMC Xilinx fast memory controller. Each OP can access the video buffer through a dedicated VFBC port; each port is connected to a 32bits FIFO. The Xilinx MPMC component allows for 8 ports, which means that up to 4 OPs can be implemented. The Y-buffer may not be necessary; it actually depends on the number of simultaneously visible objects. However if we consider $9 \mathrm{~km}$ visibility along the Y-axis, which is a very good assumption according to (Franklin, 2006) (5km max), then a 4Mbits memory is required. Regarding the Artix low cost low power device (12Mbits on-chip) this solution could be implemented on a single reconfigurable chip.

\subsection{OP Architecture}

The OP component is the main architectural contribution of the proposed design, it is described in Fig.9. Given new user attitude from the processor where positioning algorithms are implemented, each OP updates the position and the drawing of the objects it is in charge of. The CPU stores object initial coordinates and features (polygons geometry, colors and so on) in the OP local memory. The CPU can decide the load of each OP and the choices of objects to be drawn according to user priorities and field of vision. The $\mathrm{OP}$ is a strongly optimized $\mathrm{N}$ bits architecture where $\mathrm{N}$ can be decided at design time according to accuracy constraints (e.g. $\mathrm{N}=16$ bits, 11 integer and 5 rational). The design has been focused on data locality and bandwidth optimization, and the whole computation is controlled with a 223 states FSM, which is organised in 5 main steps:

1) Attitude and position data acquisition and coordinate system transformations (37 states)

2) Object scaling and $2 \mathrm{D}$ projection (27)

3) Visibility tests and vertex color computation (40)

4) Polygon shape tests and color increment computation (66)

5) Polygon Drawing (53).

Each OP implements two application-specific

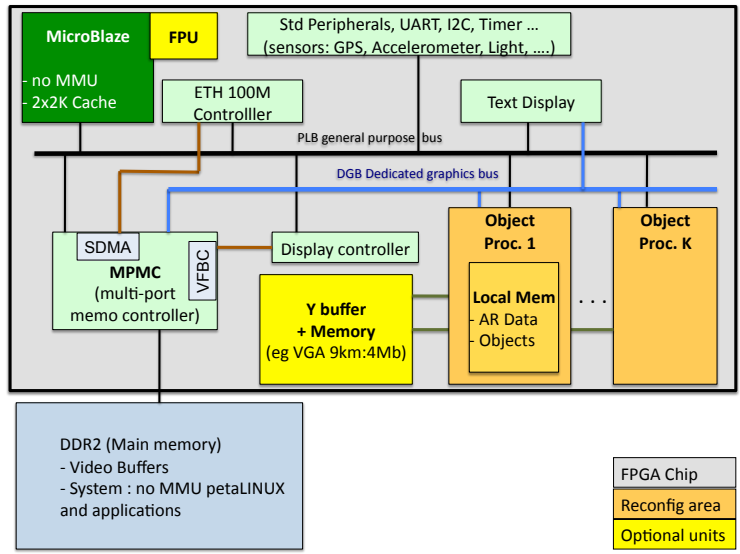

Figure 8: Heterogeneous multi-OP MPSoC architecture.

ALUs, the first one has 3 inputs and 13 specific but simple instructions (e.g. fast implementation of division algorithm or increment operations), the second one has 2 inputs and 7 instructions (e.g. decrement with zero test). The architecture also includes two multipliers, 13 general-purpose registers and a register file with 16 places. An important point regarding data bandwidth is the multiple memory accesses. Thus, the local memory can be accessed, though a 32 bit bus, simultaneously with the processing unit registers and the register file. Additionally the Y buffer bus and the RGB bus (24 bits pixel values) are also independently available. The size of the memory is also flexible (e.g. 5 objects with 20 points and 10 polygons each and $\mathrm{N}=16$ bits requires 39 Kbits). The bus controller provides $32,2 \times 16$ or $4 \times 8$ bits accesses. Finally a new component called the Incremental Pixel Shader (IPS) is introduced to apply the final step of the proposed method, which is based on horizontal and vertical colour increments jointly with the double Bresenham method. It is mainly composed of registers with $\mathrm{X}, \mathrm{Y}$ and $\mathrm{Z}$ increments, an ALU and a controller. Given Kpx and Kpz, IPS can increment polygon pixel values, this component directly computes RGB values for each pixel with 6 increment or move instructions. The complete OP specification represents 16.000 lines of original VHDL code.

The architecture is such that multiple OPs can run in parallel since each of them works with a given collection of objects. The CPU and OPs are quite independent, since they rely on shared memory communications, so that the CPU can feed OP local memories with new position data. So the architecture can be dynamically and partially reconfigured according to the number of objects and real-time constraints. If new FPGA devices can implement real low-power modes when the power consumption is negligible for unconfigured areas, then energy efficiency can still be improved. As described in Fig.8, each OP can be 
placed in a dynamically reconfigurable area. Technically speaking, this solution is viable, the critical parts lie in the implementation of the two bus interfaces (VFBC, Y-buffer) that must be isolated from the reconfigurable area. However, dynamic reconfiguration is still difficult to implement and strongly dependent on CAD vendor (Xilinx) tools support.

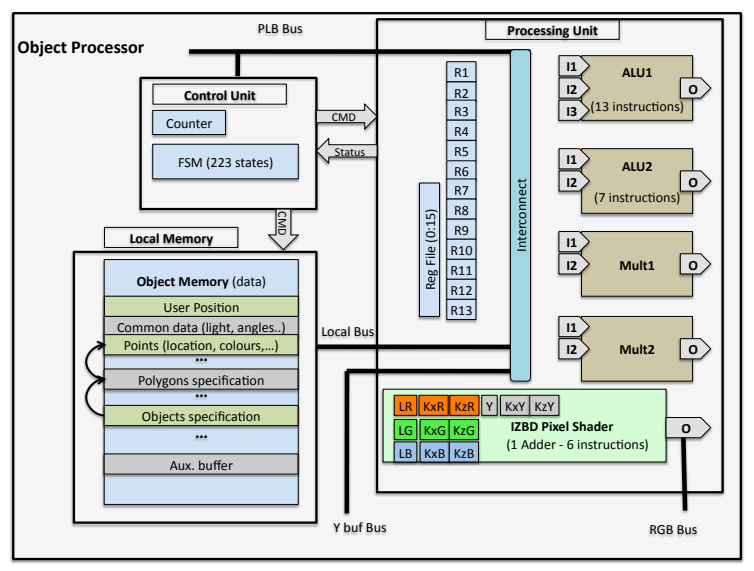

Figure 9: Graphic Object Processor Architecture.

\section{RESULTS AND DISCUSSIONS}

\subsection{Performances}

First, the whole application flow has been described with a parameterized performance model that enables the counting of operation and transfers according to 25 variables such as the display format, the number and size of objects etc. Then this model has been validated with some real profiling carried out on the target MB with and without floating point unit (FPU). The performance model for hardware implementation is straightforward since the number of steps of the FSM is fully specified. Fig.10 gives the results with the following configurations:

- "SW": fully software implementation on a MB.

- "SW+FPU": MB with a floating-point unit.

- "SW+FPU+OP": MB with FPU and one OP unit. In this example we consider a case, which goes beyond our case study requirements. Thus in this configuration, we consider 8 objects, 16 vertices and 13 polygons per object, a VGA display, an average 2D object size equal to $1 / 20$ VGA and a sensor acquisition rate of $50 \mathrm{~Hz}$ while the GPS acquisition rate is $5 \mathrm{~Hz}$. If we consider a $100 \mathrm{MHz}$ clock $(100,106$ cycles), we observe that the Positioning/Attitude control part of the application requires $70 \%$ of available cycles with the SW solution. The margin is too small to be safe within a Linux system where additional user processes are necessary. Moreover a FPU unit is of real value for matrix operations that have high precision requirements. The second solution "SW+FPU" can also be considered for the Object positioning (steps 1.1,1.2,1.3) that requires around $2 \mathrm{M}$ of cycles, but the quaternion-based rotation of objects will be too greedy (66M cycles) and would lead to a total of $80 \%$ of CPU use. As expected, the graphic part of the application is definitely out of the scope of any processor-based implementation, except for the $2 \mathrm{D}$ projection steps $(3.8 \mathrm{M})$. But combined with previous application requirements, this operation cannot be mapped on the processor. This means that the OP HW IP will handle all the graphic steps with a clear interface to the software positioning part that can feed the OP with filtered attitude and position data. The OP processor is then in charge of adapting objects drawing according to new data. Based on the previous assumptions, $40 \mathrm{M}$ cycles are required for a complete execution of the graphics part. Table 3 gives implementation of the OP HW IP on three Xilinx FPGA families: Virtex 5, 6 and Spartan 6. The last one exhibits the lowest clock frequency $(73 \mathrm{MHz})$, which means $73 \mathrm{M}$ available cycles and a consequently a use rate equal to $55 \%$. In the two other cases, the clock frequency is $120 \mathrm{MHz}$ and the use rate drops to $33 \%$. As a conclusion we observe that a viable solution is a MicroBlaze with a FPU, running an embedded Linux OS and enhanced with a HW OP IP. This architecture offers the expected performance to run augmented reality applications. We now have to check hardware implementation costs.

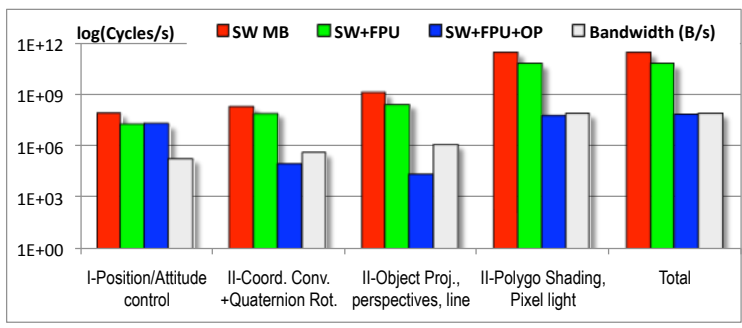

Figure 10: Performance summary considering: 8 objects, 16 vertex/Obj., 13 triangles / Obj., VGA display, average 2D object size: 1/20 VGA, sensor acquisition rate: $50 \mathrm{~Hz}$, GPS acquisition rate: $5 \mathrm{~Hz}$.

\subsection{Implementation Cost}

The OP HW IP is specified as VHDL code at RTL level. This code has been synthesised, placed and routed for three Xilinx devices. Synthesis results are given in Table 1 and projections on different FPGA devices and families are given in Table 2. The MB 
configuration implements a 4K I/D cache and usual peripheral controllers (UART, MPMC, Flash, Ethernet, VGA). Table 1 gives the number of slices and BRAM blocks required for $\mathrm{MB}+\mathrm{FPU}$ and $\mathrm{OP}$ implementation and the maximum frequency clock after place and route. We observe that a OP is half the cost of a MB+FPU and that all implementations can reach $100 \mathrm{MHz}$ except for OP on Spartan $6(73 \mathrm{MHz})$. Note also that the results don't include the Y-buffer memory, which aren't synthesized on the FPGA in these cases; however it can be implemented as a buffer in the DDR. The consequence is that the computation of hidden polygons may be interrupted a few cycles later. Table 2 gives the number of OP that may be implemented on the different targets according to the number of Slices and BRAM blocks, it also gives the ratio with the Y-buffer size considering a 16 bits depth and VGA display (4.92Mb). The used bandwidth metric takes into account all transfers between OPs and the external DDR memory including Y-buffer accesses, this metric is strongly dominated by pixel write access for updates $(>99 \%)$. Note that we assume a very high video rate equal to the sensor rate, i.e., $50 \mathrm{~Hz}$. The Xilinx memory controller (MPMC) can manage up to 8 memory ports with different protocols (FIFO, DDR, cache,...), 4 ports are used by the MB, so 4 remain available for OP accesses to the DDR. We can draw several conclusions from these results. We first observe that the smallest Virtex 5 could theoretically implement one OP but a usage of $95 \%$ of slices puts too much constraint on the routing step. However the next device (LX50) can implement two OPs. An LX110 is required to fully implement the Y-buffer on chip, such a chip can implement 4 OPs. With the next generation of Virtex, we note that the smallest device (LX75) is large enough to design a system with 4 OPS with an on-chip Y-buffer. A LX 130 can theoretically implement 48 OPS but is limited to 12 according to the memory bandwidth capacities. Virtex are expensive FPGAs while the Spartan family has been designed for low cost design that fit the context of embedded systems. We observe that a small LX25 Spartan 6 can implement 1 OP, a LX45 provides enough resources for 3 OPs. The first solution that allows for an in-chip Y-buffer is the largest Spartan that can implement 12 OP according to bandwidth constraints. So low cost solutions with high performances are possible and more can be expected. Actually, Xilinx has released a new device generation that relies on a $28 \mathrm{~nm}$ technology in 2011. Impressive power optimizations are available with this new generation, especially with the low-cost, low-power Artix 7 family. We didn't have the possibility to run synthesis on this new target, so we made a rough es- timation based on the number of Slices and BRAM according to Spartan 6 results. It appears that a small XC7A30T could implement a one-OP solution and a medium-range XC7A100T would be large enough to design a 8-OP solution including a Y-buffer on chip. Finally the new Zynq architecture, based on a ARM dual-core Cortex A9 combined with FPGA on a single chip, offers interesting perspectives. The datasheets show that the programmable part is equivalent to an XC7A50T. It means that the positioning/control part of the application can be mapped on one core and 5 OPS on the programmable area.

\section{CONCLUSIONS}

Few research works have been conducted in the domain of embedded system design for Mobile Augmented Reality applications in the context of emerging light see-through HMD. In this project we have specified and designed a complete system according to strong size constraints. The solution that has been developed is flexible and fits with upcoming low-cost, low-power FPGAs. The approach has deliberately been focused on standard protocols and interfaces; it can be interconnected with usual inertial sensor and communication peripherals. This work results in a new approach for the design of AR-specific embedded and reconfigurable systems with four main contributions. This is the choice and the full specification of a gyroscope-free set of algorithms for position and attitude estimation, this solution relies on the association and the adaptation, to the AR domain, of different previous contributions. It demonstrates that a standard $100 \mathrm{MHz}$ Softcore can both handle Linux and motion filtering/estimation algorithms. A new embedded system architecture is introduced, it relies on a fast and simple Object Processor (OP) optimized for the domain of mobile AR. The OP implements a new pixel rendering method (IPS) implemented in hardware and that takes full advantage of Open-GL ES light model recommendation. Finally the whole architecture has been implemented on various FPGA targets, the results demonstrate that expected performances can be reached and that a low-cost FPGA can implement multiple OP.

\section{ACKNOWLEDGEMENTS}

This work has been supported by DGA (french defense department) and has greatly benefited from discussions with Dr. John Williams about system architecture and Linux implementation on FPGA. 
Table 1: OP implementation: Synthesis Results.

\begin{tabular}{|l|l|l|l|l|l|l|l|l|l|}
\hline & \multicolumn{3}{|l|}{ Virtex 5 } & \multicolumn{2}{l|}{ Virtex 6 } & \multicolumn{3}{l|}{ Spartan 6 } \\
\hline & Slices & Bram18 & F & Slices & Bram18 & F & Slices & Bram18 & F \\
\hline MB+FPU & 3305 & 20 & $120 \mathrm{MHz}$ & 2741 & 40 & $150 \mathrm{MHz}$ & 1842 & 8 & $100 \mathrm{MHz}$ \\
HW IP OP & 1231 & 2 & $120 \mathrm{MHz}$ & 1048 & 2 & $120 \mathrm{MHz}$ & 1233 & 2 & $73 \mathrm{MHz}$ \\
\hline
\end{tabular}

Table 2: Device Choice and Opportunities.

\begin{tabular}{|c|c|c|c|c|c|c|c|c|c|}
\hline & Slices & Bram18 & $\begin{array}{l}\mathrm{Nb} \text { of } \\
\mathrm{MB}\end{array}$ & $\begin{array}{l}\mathrm{Nb} \text { of } \\
\mathrm{OP}\end{array}$ & $\begin{array}{l}\text { used } \\
\text { slices }(\%)\end{array}$ & $\begin{array}{l}\text { used } \\
\text { Bram }(\%)\end{array}$ & $\begin{array}{l}\text { \% Bram } \\
+ \text { Y-buff }\end{array}$ & $\begin{array}{l}\text { Nb of } \\
\text { MPMC }\end{array}$ & $\begin{array}{l}\text { used } \\
\text { BW }(\%)\end{array}$ \\
\hline \multicolumn{10}{|l|}{ Virtex 5} \\
\hline Min(LX30) & 4800 & 64 & 1 & 1 & 0.95 & 0.34 & 3.90 & 1 & 0.08 \\
\hline LX50 & 7200 & 96 & 1 & 2 & 0.80 & 0.25 & 2.62 & 1 & 0.15 \\
\hline LX85 & 12960 & 192 & 1 & 4 & 0.63 & 0.15 & 1.33 & 1 & 0.30 \\
\hline \multirow[t]{2}{*}{ LX110 } & 17280 & 256 & 1 & 8 & 0.76 & 0.14 & 1.03 & 2 & 0.60 \\
\hline & 17280 & 256 & 1 & 4 & 0.48 & 0.11 & 1.00 & 1 & 0.30 \\
\hline LX155 & 24560 & 384 & 1 & 12 & 0.74 & 0.11 & 0.71 & 2 & 0.90 \\
\hline $\operatorname{Max}(\mathrm{LX} 330)$ & 51840 & 576 & 1 & 12 & 0.35 & 0.08 & 0.47 & 2 & 0.90 \\
\hline \multicolumn{10}{|l|}{ Virtex 6} \\
\hline Min(LX75) & 11640 & 312 & 1 & 4 & 0.60 & 0.15 & 0.88 & 1 & 0.30 \\
\hline LX130 & 56880 & 528 & 1 & 12 & 0.27 & 0.12 & 0.55 & 2 & 0.90 \\
\hline \multicolumn{10}{|l|}{ Spartan 6} \\
\hline LX25 & 3758 & 52 & 1 & 1 & 0.82 & 0.19 & 4.57 & 1 & 0.08 \\
\hline LX45 & 6822 & 116 & 1 & 3 & 0.81 & 0.12 & 2.08 & 1 & 0.23 \\
\hline LX75 & 11662 & 172 & 1 & 6 & 0.79 & 0.12 & 1.44 & 2 & 0.45 \\
\hline $\operatorname{Max}(\mathrm{LX} 100)$ & 15822 & 268 & 1 & 8 & 0.74 & 0.09 & 0.94 & 2 & 0.60 \\
\hline \multicolumn{10}{|c|}{ Projection on Artix 7 based on Spartan 6} \\
\hline \multirow[t]{2}{*}{ XC7A30T } & 5250 & 104 & 1 & 1 & 0.59 & 0.10 & 2.28 & 1 & 0.08 \\
\hline & 5250 & 104 & 1 & 2 & 0.82 & 0.12 & 2.30 & 1 & 0.15 \\
\hline XC7A50T & 150 & 150 & 1 & 4 & 0.83 & 0.11 & 1.62 & 1 & 0.30 \\
\hline ХC7A100T & 15850 & 270 & 1 & 4 & 0.43 & 0.06 & 0.90 & 1 & 0.30 \\
\hline ХС7А100T & 15850 & 270 & 1 & 8 & 0.74 & 0.09 & 0.93 & 2 & 0.60 \\
\hline
\end{tabular}

\section{REFERENCES}

Benkrid, K. (2010). Reconfigurable computing in the multicore era. In Int. Workshop on Highly Efficient Accelerators and Reconfigurable Technologies (HEART).

Bijker, J. and Steyn, W. (2008). Kalman filter configurations for a low-cost loosely integrated inertial navigation system on an airship. Control Engineering Practice, 16(12):1509 - 1518.

Franklin, M. (2006). The lessons learned in the application of augmented reality. In RTO Human Factors and Medicine Panel (HFM) Workshop, West Point, NY, USA. NATO.

Gebre-Egziabher, D., Elkaim, G. H., Powell, J. D., and Parkinson, B. W. (2000). A gyro-free quaternionbased attitude determination system suitable for implementation using low cost sensors. In IEEE Position Location and Navigation Symposium, pages 185-192.

Guo, P.-F., Qiu, H., Yang, Y., and Ren, Z. (2008). The soft iron and hard iron calibration method using extended kalman filter for attitude and heading reference system. In Position Location and Navigation Symp. (PLANS).

Kim, K. H., Lee, J. G., and Park, C. G. (2009). Adaptive two-stage extended kalman filter for a fault-tolerant ins-gps loosely coupled system. Aerospace and Electronic Systems, IEEE Trans. on, 45(1):125-137.

Kingyens, J. and Steffan, J. G. (2011). The potential for a gpu-like overlay architecture for fpgas. International Journal of Reconfigurable Computing, 2011.
Koo, W., Chun, S., Sung, S., Lee, Y. J., and Kang, T. (2009). In-flight heading estimation of strapdown magnetometers using particle filters. In National aerospace \& electronics IEEE conference (NAECON).

Li, D., Landry, R. J., and Lavoie, P. (2008). Low-cost mems sensor-based attitude determination system by integration of magnetometers and gps: A real-data test and performance evaluation. In IEEE Position Location and Navigation Symposium.

Lingley, A., Ali, M., Liao, Y., Mirjalili, R., Klonner, M., Sopanen, M., Suihkonen, S., Shen, T., Otis, B. P., Lipsanen, H., and Parviz, B. A. (2011). A single-pixel wireless contact lens display. Journal of Micromechanics and Microengineering, 21(12):125014.

Nasiri, S. (2010). A critical review of mems gyroscopes technology and commercialization status. Technical report, InvenSense, http://invensense.com/.

Shin, E.-H. and El-Sheimy, N. (2004). An unscented kalman filter for in-motion alignment of low-cost imus. In Position Location and Navigation Symposium, 2004. PLANS 2004, pages 273-279.

Waegli, A., Skaloud, J., TomÃ (C), P., and Bonnaz, J.-M. (2007). Assessment of the Integration Strategy between GPS and Body-Worn MEMS Sensors with Application to Sports. In ION-GNSS 2007.

Zhu, R., Sun, D., Zhou, Z., and Wang, D. (2007). A linear fusion algorithm for attitude determination using low cost mems-based sensors. Measurement, 40(3). 